\title{
Ventilation Adjustment in ECT During COVID-19: Voluntary Hyperventilation is an Effective Strategy
}

\author{
Aida de Arriba-Arnau $\mathbb{D}^{1,2}$ \\ Antònia Dalmau Llitjos ${ }^{3}$ \\ Virginia Soria $1,2,4$ \\ Javier Labad 2,5,6 \\ José Manuel Menchón iD 1,2,4 \\ Mikel Urretavizcaya ${ }^{1,2,4}$ \\ 'Department of Psychiatry, Bellvitge \\ University Hospital-ICS, Bellvitge \\ Biomedical Research Institute (IDIBELL), \\ Neurosciences Group - Psychiatry and \\ Mental Health. L'Hospitalet de Llobregat, \\ Barcelona, Spain; ${ }^{2}$ Centro de Investigación \\ Biomédica en Red de Salud Mental \\ (CIBERSAM), Carlos III Health Institute, \\ Barcelona, Spain; ${ }^{3}$ Department of \\ Anesthesiology, Reanimation and Pain \\ Clinic, Bellvitge University Hospital-ICS, \\ Universitat de Barcelona (UB), Barcelona, \\ Spain; ${ }^{4}$ Department of Clinical Sciences, \\ School of Medicine, Universitat de \\ Barcelona (UB), Barcelona, Spain; \\ ${ }^{5}$ Department of Mental Health, Consorci \\ Sanitari del Maresme, Mataró, Spain; ${ }^{6}$ Parc \\ Taulí, Institut d'Investigació i Innovació Parc \\ Taulí (I3PT), Barcelona, Spain
}

Purpose: Airway management is a key objective in adapted electroconvulsive therapy (ECT) protocols during the COVID-19 pandemic to prevent infection. The objective of this study was to describe the effectiveness of a modified ventilation procedure designed to reduce aerosol-generating bag-mask ventilation (BMV) and isolate possible droplets while maintaining adequate respiratory gas values in ECT sessions.

Materials and Methods: This prospective study analyzed the results of the modified protocol applied over a month. Adaptations entailed preoxygenation and extension of the voluntary hyperventilation (VHV) time for two minutes before anesthesia induction, asking patients to hyperventilate with oxygen therapy via nasal cannula and while wearing a face mask. Thereafter, vigorous hyperventilation was avoided, and patients were only assisted with tightly sealed BMV until emergence from anesthesia, isolating the ventilation by using a single-use plastic device. Oxygen saturation $\left(\mathrm{SpO}_{2}\right)$ and transcutaneous partial pressure of carbon dioxide $\left(\mathrm{TcPCO}_{2}\right)$ were recorded throughout the session.

Results: The study included 74 sessions of bilateral ECT with the modified ventilation protocol in 15 subjects. After VHV, the mean $\mathrm{SpO}_{2}$ increase was $2.12 \pm 2.14 \%$, and the mean $\mathrm{TcPCO}_{2}$ decrease was $4.05 \pm 2.98 \mathrm{mmHg}$. $\mathrm{TcPCO}_{2}$ values at the moment of stimulus administration were $2.22 \pm 3.07 \mathrm{mmHg}$ below pre-ECT values. The mean EEG seizure was 38.70 $\pm 17.03 \mathrm{~s}$, and postictal suppression was $68.31 \pm 34.58 \%$ and $2.13 \pm 0.75$ on a $0-3$ scale. Brief desaturation $\left(\mathrm{SpO}_{2}<90\right)$ of $4-5$ seconds duration was observed in 4 sessions.

Conclusion: This modified ventilation protocol was effective during COVID-19, and it did not elicit significant side effects. In addition to avoiding vigorous BMV, it induced moderate hypocapnia, which has been tied to seizure optimization and less hypercapnia during the apnea period.

Keywords: electroconvulsive therapy anesthesia, ECT, airway, bag-mask ventilation, oxygenation, self-hyperventilation, hypocapnia

\section{Introduction}

The quick overwhelming impact that the COVID-19 pandemic produced on many countries' health care systems initially decreased the activity of some electroconvulsive therapy (ECT) units. Many physicians have claimed that this treatment should be administered to patients who need it despite current circumstances, ${ }^{1-3}$ and there is a clear and universal consensus that ECT is a critical and essential treatment. $^{4,5}$

There are inherent aspects of ECT that increase its risk during the pandemic. Many patients are older adults who are at higher risk of COVID-19 than the general population and have comorbidities, ${ }^{5}$ the staff usually rotate, and the ECT procedure
Correspondence: Mikel Urretavizcaya Sarachaga Department of Psychiatry. Bellvitge University Hospital - ICS, IDIBELL, CIBERSAM; UB Feixa Llarga s/n, 08907, L'Hospitalet de Llobregat, Barcelona, Spain Tel +34932607922

Fax +34932607658

Email murretavizcaya@bellvitgehospital.cat 
under anesthesia involves airway management requirements. ${ }^{6}$ Additionally, ventilation during the treatment facilitates aerosol dispersion, ${ }^{5,7}$ and COVID is transmitted through respiratory droplets. Hence, the ECT procedure has been rapidly adapted with changes intended to maintain safety for patients and staff. ${ }^{5}$

One of the key ECT-specific procedure changes in clinical practice to minimize infection exposure is airway management. Several modifications in terms of ventilation during the ECT application technique have been proposed, but unfortunately, the impact of many of these interventions on ECT results has not been studied.

Hyperventilation is widely recommended in ECT guidelines because it is a simple and effective strategy for improving seizure quality and patient safety. ${ }^{8}$ However, in the setting of COVID-19, some authors recommend avoiding or minimizing hyperventilation because it is usually performed by manual bag-mask ventilation (BMV). ${ }^{6} \mathrm{BMV}$ is an aerosol-generating or droplet dispersion procedure $5,9,10$ due to its poor airway seal, which poses the greatest exposure risk to patients and staff. ${ }^{5}$ Other authors proposed improving preoxygenation instead, ${ }^{1}$ with a regular or nonrebreather mask for several minutes pretreatment ${ }^{5}$ and avoiding BMV unless severe desaturation occurred, ${ }^{10}$ but some patients still require $\mathrm{BMV}$, $^{5,10}$

The use of procedures that allow a good airway seal through more invasive methods has also been recommended by some authors. However, using tracheal intubation in all patients may prolong the ECT procedure and induce gagging and coughing. ${ }^{11}$ Others recommended the use of supraglottic airways (establishing a closed circuit system and extubating under a plastic tent) because its insertion and removal limits aerosolization more than tracheal intubation. ${ }^{7}$ It has also been proposed that ECT should be administered in an operating room setup with negative pressure, if available, ${ }^{9}$ especially if the patient is ill with COVID-19, but this is not always available in all centers. ${ }^{5}$

The aim of this study was to describe the effectiveness of a modification to the ventilation procedure protocol proposed during the COVID-19 pandemic that addresses the need to reduce aerosol-generating $\mathrm{BMV}$ and isolate possible droplets. We hypothesize that by implementing voluntary hyperventilation, hypocapnia and adequate oxygenation can be optimized.

\section{Materials and Methods}

We prospectively collected data from the sessions performed in the ECT Unit of the Bellvitge University
Hospital during June 2020 using a ventilation protocol that addresses the need to reduce aerosol-generating bagmask ventilation and isolate possible droplets during the COVID-19 pandemic. $^{12}$ We analyzed the effectiveness of this protocol in reaching adequate values of respiratory gases in ECT sessions and adequate seizures. All patients provided written informed consent, and the study was approved by the Bellvitge University Hospital Clinical Research Ethics Committee. All procedures were performed according to the Declaration of Helsinki.

Bilateral ECT was applied using a Thymatron System IV device (Somatics, LLC, Lake Bluff, III). Anesthesia was induced with thiopental $(1.5-2.5 \mathrm{mg} / \mathrm{kg})$ or etomidate $(0.1-0.3 \mathrm{mg} / \mathrm{kg})$, and succinylcholine $(\sim 0.5 \mathrm{mg} / \mathrm{kg})$, adapting dosages to patients' needs. Concomitant psychotropic medications were maintained during the treatment course.

The standard ECT procedure was adapted by a multidisciplinary team covering mainly prevention of infection risk and the airway management maneuvers involved in modern ECT. A specific hospital protocol (Electroconvulsive therapy procedure during the COVID19 pandemic (v15.05.2020)), which followed the recommendations of international (International Society of ECT and Neurostimulation (ISEN) ${ }^{11}$ ) and Spanish societies (the Spanish Society of Biological Psychiatry (SEPB) general recommendations to adapt the practice of electroconvulsive therapy during the COVID-19 pandemic ${ }^{6}$ ), local COVID-19 guidelines, and the current literature, ${ }^{7,9,10,13}$ was developed. This protocol was approved by the hospital's quality department and was applied in all patients treated with ECT.

The ECT unit applied structural adjustments to keep a safe distance and a separated clean circuit patient flow; organizational changes included keeping minimum staff during the treatment; controlled appointment time; a decreased number of patients; natural ventilation of the room for 15-30 minutes to allow more air recirculation between patients and cleaning with disinfectant wipes between patients; and a comprehensive intensive room cleaning protocol applied at the end of the day sessions or between outpatients' and inpatients' sessions.

Before entering the ECT Unit, patients underwent a screening for potential contact with COVID-19 patients, reported clinical symptoms, vital signs, respiratory rate, $\mathrm{SpO}_{2}$, temperature control, negative polymerase chain reaction (PCR) testing for SARS-CoV-2 (in the last 48 h), and absence of respiratory symptoms. Patients wear protective surgical face masks at all times inside the ECT 
unit, ${ }^{14}$ and the staff also wears the required personal protective equipment (PPE).

The half-age method of stimulation ${ }^{15}$ was used to avoid titration restimulations and possible lengthening of the session duration and the time the patient was not being ventilated, and succinylcholine was optimized at the lower dose range for a quicker return to spontaneous breathing after the seizure. ${ }^{5}$

During the treatment, a single-use standard nasal cannula was used for oxygenation, ${ }^{9}$ and single-use adherent stimulus electrodes were used to prevent contamination and cross-infection and maintain distance. A disinfected mouth guard that allowed ventilation through itself was appropriately placed before the patient was anesthetized. Further manipulation of the airway, such as the introduction of the Guedel cannula, was avoided when possible. Additionally, a single-use disposable plastic cover with an adaptable hole to connect the disinfected bag mask and antimicrobial air filter was used to prevent aerosol spreading.

Changes to the usual ventilation procedure were applied to minimize aerosol generation and droplet dispersion while maintaining adequate respiratory gases and seizure parameters. The modifications included preoxygenation with a single-use nasal cannula with oxygen at $\approx 4 \mathrm{~L} / \mathrm{min}$ below the protective surgical face mask that patients wore. Then, to decrease $\mathrm{TcPCO}_{2}$ basal values, voluntary hyperventilation (VHV) was performed asking the patient to self-hyperventilate for 2 minutes before anesthetic induction. Afterwards, once the patient was asleep, the waterproof plastic cover was in place to isolate ventilation and the airways. The protocol avoids vigorous BMV manual hyperventilation and mouth manipulation if possible, performing ventilation through the mouth guard if needed and maintaining oxygenation and manual ventilation assistance with a tight sealed BMV under the plastic tent until the patient recovers spontaneous breathing. ${ }^{12}$

Patients underwent continuous monitoring throughout all sessions using assessments of electrocardiogram, blood pressure, oxygen saturation $\left(\mathrm{SpO}_{2}\right)$ by pulse oximetry (Datex Engstrom AS/3 ${ }^{\mathrm{TM}}$ (Datex-Engström, Helsinki, Finland) anesthesia monitor), and transcutaneous partial pressure of carbon dioxide $\left(\mathrm{TcPCO}_{2}\right)$ using a noninvasive estimation of partial arterial $\mathrm{CO}_{2}$ pressure $\left(\mathrm{SenTec}^{\circledR}\right.$ Digital Monitoring System (SenTec AG, Therwil, Switzerland)).

$\mathrm{TcPCO}_{2}$ was continuously monitored throughout all sessions, and $\mathrm{TcPCO}_{2}$ values were specifically recorded at each phase of ventilation management: T1, basal values of $\mathrm{TcPCO}_{2}$ before the treatment session; $\mathrm{T} 2$, during preoxygenation and at the beginning of voluntary hyperventilation; T3, at the end of voluntary hyperventilation and beginning of anesthesia induction; T4, minimum value achieved after the 2-min voluntary hyperventilation; T5, after manual ventilation assistance performed avoiding vigorous hyperventilation, once the patient is asleep; T6, values at the moment of ECT stimulus delivery; T7, values at the end of the seizure; T8, post-ECT period value after patient's recovery of autonomous breathing and consciousness and at the time of transferring the patient to the recovery room.

The main values considered in the study were $\mathrm{T} 1, \mathrm{~T} 4$, T6, and T8 (see Figure 1).

Seizure characteristics were determined by 2-channel electroencephalogram (EEG), duration and postictal suppression index (PSI) were provided by the Thymatron device, and the motor and EEG seizure duration plus the postictal suppression rating (PSIr) using a $0-3$ scale $^{16,17}$ were determined visually by a psychiatrist with expertise in ECT.

Statistical analyses were performed using SPSS v19.0 (SPSS, an IBM company, Chicago, Illinois, USA); $\mathrm{R}^{18}$ and R studio. ${ }^{19}$ The statistical significance was set at $\mathrm{p}<0.05$ (bilateral).

To study the $\mathrm{TcPCO}_{2}$ dynamics during the sessions that is presented in Figure 1, R and $\mathrm{R}$ studio were used for exploring longitudinal changes in $\mathrm{TcPCO}_{2}$ after VHV. The lme4 package was used for fitting linear mixed effect models with the maximum likelihood method. Fixed effects were considered for the number of ECT sessions, time, and cooperation with the VHV procedure. Random effects were assigned to each participant. Time and cooperation variables were defined as factors, and the interaction between time and cooperation was tested. The reference categories for these two factors were baseline time (T1) and lack of cooperation. With this decision, the model allows to estimate the changes in $\mathrm{TcPCO} 2$ from baseline time and to consider whether the change at each time depends upon the cooperation of the patient with the VHV procedure (tested with an interaction).

Pearson correlations were used to analyse the relationships between ventilation parameters and seizure characteristics (duration and postictal suppression).

\section{Results}

A total of 74 sessions in 15 consecutive patients were performed during the study period. In seven of these 


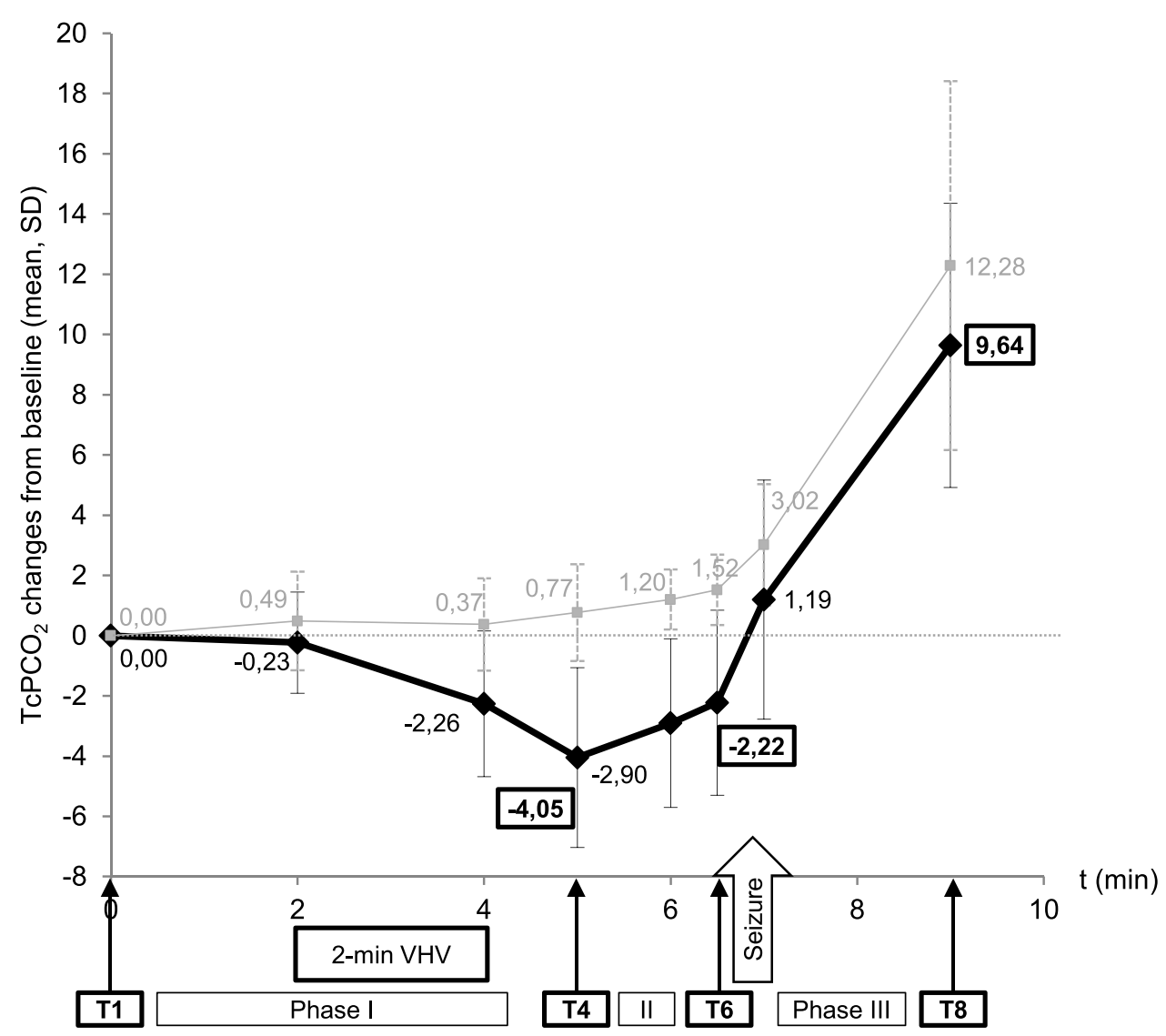

Figure I Patterns of change in transcutaneous partial pressure of carbon dioxide values during the ECT procedure.

Note: $\bullet$ Values obtained using the ventilation procedure during COVID-19 at different measurement time points in the ECT session. Values from sessions where patients were unable to cooperate with the 2-minute voluntary hyperventilation. Relevant points in time and phases in the ECT session according to the ventilation procedure: TI, baseline values before treatment; T4, minimum value achieved after the 2-min voluntary hyperventilation; T6, at the moment of ECT stimulus application; T8, after ECT. Phase I, preoxygenation and voluntary hyperventilation until anesthesia induction; Phase II, after anesthesia induction and until stimulus delivery, manual ventilation assistance was performed avoiding vigorous hyperventilation; Seizure; Phase III, patient's recovery of autonomous breathing and consciousness.

Abbreviations: ECT, electroconvulsive therapy; t, time in minutes; $\mathrm{TcPCO}_{2}$, transcutaneous carbon dioxide measures; VHV, voluntary hyperventilation during approximately 2 minutes.

sessions, patients were unable to cooperate with the proposed VHV maneuvers due to their clinical status.

The characteristics of the sample, the ventilation parameters, and the seizures obtained while performing this ventilation approach are presented in Table 1.

After VHV, the mean $\mathrm{SpO}_{2}$ increase was $2.12 \pm 2.14 \%$, and the mean decrease in $\mathrm{TcPCO}_{2}$ from basal values was $4.05 \pm 2.98 \mathrm{mmHg}$. $\mathrm{CO}_{2}$ dynamics during the sessions are presented in Figure 1.

After anesthetic induction, $\mathrm{TcPCO}_{2}$ values increased because vigorous hyperventilation was avoided to prevent aerosol generation. Nevertheless, a mean reduction from baseline values of $2.22 \pm 3.07 \mathrm{mmHg}$ in $\mathrm{TcPCO}_{2}$ was still maintained at the moment of stimulus administration.

Changes in $\mathrm{TcPCO} 2$ during the ECT procedure by cooperation with the VHV procedure are described in
Figure 1. The linear mixed model analysis testing the contribution of time and cooperation shows that changes at $\mathrm{T} 7$ and $\mathrm{T} 8$ (after the seizure) are significant from baseline independently of the cooperation, although there are significant differences, with lower TcPCO2 concentrations, in the group of participants who cooperated with the VHV, at times T4 and T5 (before the seizure). This analysis was adjusted for the number of ECT sessions. The results of the fixed effects are shown in Table S1.

EEG duration correlated with the oxygen increase at the ECT stimulus delivery (T6) $(\mathrm{r}=0.41, \mathrm{p}=0.001)$. PSI was correlated with the $\mathrm{TcPCO}_{2}$ decrease at $\mathrm{T} 4$ $(\mathrm{r}=0.31, \mathrm{p}=0.031)$ and $\mathrm{TcPCO}_{2}$ values after VHV at $\mathrm{T} 4(\mathrm{r}=-0.26, \mathrm{p}=0.037)$ and the ECT stimulus delivery (T6) $(r=-0.33, p=0.009)$. 
Table I Ventilation Parameters and Demographic, Clinical, and ECT Characteristics of the Study Population

\begin{tabular}{|c|c|}
\hline Variables & \\
\hline \multicolumn{2}{|l|}{ Patients' characteristics } \\
\hline Age (years), mean (SD) & $67.00(14.8)$ \\
\hline Female, \% (n) & $73.33 \%(11 / 15)$ \\
\hline Body Mass Index, mean (SD) & $27.02(6.37)$ \\
\hline ASA I, \% (n) & $6.7 \%(1 / 15)$ \\
\hline ASA II, \% (n) & $73.3 \%(11 / 15)$ \\
\hline ASA III, \% (n) & $20.0 \%(3 / 15)$ \\
\hline Smokers, \% (n) & $40 \%(6 / 15)$ \\
\hline \multicolumn{2}{|l|}{ Psychiatric diagnose } \\
\hline Major depressive disorder,\% ( $n$ ) & $56.67 \%(7 / 15)$ \\
\hline Bipolar disorder, \% (n) & $26.67 \%(4 / 15)$ \\
\hline Schizoaffective disorder, \% (n) & $20.00 \%(3 / 15)$ \\
\hline Schizophrenia, \% (n) & $6.67 \%(1 / 15)$ \\
\hline \multicolumn{2}{|l|}{ Current episode } \\
\hline Psychotic symptoms, \% (n) & $26.67 \%(4 / 15)$ \\
\hline Melancholic symptoms, \% (n) & $60.00 \%(9 / 15)$ \\
\hline Catatonic symptoms, \%(n) & $13.33 \%(2 / 15)$ \\
\hline \multicolumn{2}{|l|}{ ECT sessions characteristics } \\
\hline \multicolumn{2}{|l|}{ ECT parameters } \\
\hline Stimulus intensity (\%), mean (SD) & $56.34 \%(25.77)$ \\
\hline I ms pulse width, $\%$ & $23.88 \%$ \\
\hline $0.75 \mathrm{~ms}$ pulse width, \% & $2.99 \%$ \\
\hline 0.5 ms pulse width, $\%$ & $67.16 \%$ \\
\hline 0.25 ms pulse width, $\%$ & $2.99 \%$ \\
\hline Mean ASTI (s), mean (SD) & $175(45.27)$ \\
\hline Motor seizure duration (s), mean (SD) & $27.19(17.9)$ \\
\hline EEG seizure duration (s), mean (SD) & $38.70(17.03)$ \\
\hline PSI (\%), mean (SD) & $68.31(34.58)$ \\
\hline PSI (0-3 scale), mean (SD) & $2.13(0.75)$ \\
\hline \multicolumn{2}{|l|}{ Anaesthesia parameters } \\
\hline Thiopental, \% & $80.60 \%$ \\
\hline Etomidate, \% & $19.40 \%$ \\
\hline \multicolumn{2}{|l|}{ Ventilation parameters } \\
\hline \multicolumn{2}{|l|}{$\mathrm{TcPCO}_{2}$} \\
\hline Baseline (TI), mean (SD) & $37.58(5.90)$ \\
\hline Post VHV (T4), mean (SD) & $33.86(6.5 \mathrm{I})$ \\
\hline At the time of stimulus application (T6), mean(SD) & $35.58(7.10)$ \\
\hline Post ECT (T8), mean (SD) & $47.41(8.13)$ \\
\hline \multicolumn{2}{|l|}{$\mathrm{SpO}_{2}$} \\
\hline Baseline (TI), mean (SD) & $97.26(2.17)$ \\
\hline Post VHV (T4), mean (SD) & $99.48(0.70)$ \\
\hline At the time of stimulus application (T6), mean (SD) & $99.07(0.50)$ \\
\hline Post ECT (T8), mean (SD) & $97.83(3.16)$ \\
\hline
\end{tabular}

Notes: Relevant points in time according to the ventilation procedure: TI, basal values before the treatment; T4, after voluntary hyperventilation; T6, at the time of stimulus delivery; T8, after ECT.

Abbreviations: ASA, American Society of Anesthesiologists physical status classification system; ASA I, normal healthy patients; ASA II, patients with mild systemic disease; ASA III, patients with severe systemic disease; ASTI, time interval from the beginning of anesthesia administration until ECT stimulus application; EEG, electroencephalogram; ms, milliseconds; PSI, postictal suppression index; SD, standard deviation; $\mathrm{SpO}_{2}$, oxygen saturation; $\mathrm{TcPCO}_{2}$, transcutaneous carbon dioxide measures; VHV, voluntary hyperventilation during approximately 2 minutes. 
In 4 sessions, $\mathrm{SpO}_{2}<90 \%$ was observed after the seizure, and these values recovered 4-5 seconds after restarting ventilation support. All 4 instances occurred in patients with baseline $\mathrm{SpO}_{2}<95 \%$ and long seizures; all were smokers, and one of these patients was obese. No other complications were noted.

Finally, in the 7 sessions where the patients were unable to cooperate with the 2-minute $\mathrm{VHV}$, the mean post-ECT $\mathrm{SpO}_{2}$ was $95.57 \%$ and $\mathrm{TcPCO}_{2}$ monitoring showed higher values than baseline after anesthesia induction (Figure 1).

Additionally, the mean seizure duration was shorter (motor 19.14 $\pm 6.51 \mathrm{~s}$ and EEG $27.86 \pm 6.33 \mathrm{~s}$ ) than in the sessions of patients who cooperated in VHV (analyzed in Table 1), while the PSI was similar.

\section{Discussion}

The results of this modified ventilation protocol, including preoxygenation and 2-minute VHV, while avoiding vigorous BMV hyperventilation, were analyzed. These changes in the ventilation procedure were implemented due to the COVID-19 pandemic and effectively induced moderate hypocapnia without eliciting significant side effects.

The mean $\mathrm{TcPCO}_{2}$ decreases after two minutes of VHV $(-4.05 \mathrm{mmHg})$ and almost doubled the values of the oneminute VHV tested in a previous protocolized hyperventilation procedure $(-2.61 \mathrm{mmHg}){ }^{20}$ This finding reinforces the notion that VHV performed actively by the patient before anesthesia induction is an important maneuver to decrease $\mathrm{TcPCO}_{2}{ }^{20}$ and suggests that a longer duration of VHV performance might further reduce the achieved $\mathrm{TcPCO}_{2}$ values. In standard electroencephalography recordings, VHV for longer periods (usually approximately 3 minutes) was reported as a safe widespread epileptogenic activation maneuver for seizure provocation without significant side effects. ${ }^{21}$ Additionally, VHV has been allowed in EEG recommendations for COVID-19negative patients or when the absence of seizures is suspected despite no prior testing being available. ${ }^{22}$

In our study, a reduction of $2.22 \mathrm{mmHg}$ from baseline was maintained until the stimulus application moment, with similar values to the 2.18 decrease reached at $\mathrm{T} 3$ in the previous PHV procedure. ${ }^{20}$ This decrease in approximately 2 points from the patient's basal values was associated with reduced hypercapnia and lower $\mathrm{TcPCO}_{2}$ over the whole session, as well as increased seizure duration and correlation with seizure quality indexes. ${ }^{20}$

Furthermore, because some sites use different lengths of preoxygenation and others use patients self-hyperventilation, and since it has been proposed that these maneuvers may allow for the elimination of BMV in some cases, ${ }^{5}$ the present study findings are reassuring and illustrate the effectiveness of these maneuvers in everyday clinical ECT practice in terms of ventilation gases and seizure parameters.

Desaturation can occur during ECT, especially in patients with risk factors (obesity, ${ }^{10,23,24}$ high seizure length, ${ }^{23}$ baseline respiratory compromise ${ }^{10}$ ), and ventilation management is important to prevent this. Patient positioning, ventilation maneuvers, airway devices, and neuromuscular blocking agent recommendations have been reported to minimize desaturation incidence in morbidly obese patients. ${ }^{24}$ Using our study's proposed ventilation approach, $\mathrm{SpO}_{2}<90 \%$ occurred in only $5.9 \%$ of sessions. However, in another recent study where 3-5 minute preoxygenation was performed with a nonrebreather mask while avoiding BMV hyperventilation before the stimulus, $\mathrm{SpO}_{2}$ decreased below $85 \%$ during the apneic period in $51 / 106(48.1 \%)$ of the patients, and BMV was required to recover adequate saturations. ${ }^{10}$ Of note, $\mathrm{CO}_{2}$ was not measured, so the incidence of hypercapnia could not be quantified, but EEG seizure duration was found to be slightly reduced. ${ }^{10}$

Some limitations of this study merit discussion. The study described the results of the modified ECT protocol in our unit; however, the changes in ventilation procedures applied are easy and replicable in other centers, and they are applicable even if $\mathrm{CO}_{2}$ monitoring is not available. Analyses were exploratory and could be underpowered because of the relatively small sample size, and due to the observational design there was not a proper comparison group.

\section{Conclusion}

Our findings suggest that preoxygenation and 2 minutes of VHV is useful and well tolerated during the COVID-19 pandemics because, in addition to minimize BMV hyperventilation, it still induces moderate hypocapnia, which has been tied to seizure optimization and less hypercapnia during the apnea period in ECT sessions. Additionally, the results strengthen the importance of preoxygenation ${ }^{24}$ and support the role of $\mathrm{VHV}$, the impact of ventilation in gas dynamics during ECT sessions, ${ }^{20,25}$ and the relevance of $\mathrm{CO}_{2}$ and $\mathrm{O}_{2}$ values in the obtained seizure characteristics. ${ }^{8}$

\section{Acknowledgments}

We appreciate the participation from all the patients, their relatives and the staff from the ECT Unit of Bellvitge University Hospital. We also thank the CERCA Programme/ Generalitat de Catalunya for institutional support. 


\section{Disclosure}

Dr José Manuel Menchón reports personal fees from Janssen, personal fees from AbBiotics, outside the submitted work. The authors report no other conflicts of interest in this work.

\section{References}

1. Sienaert P, Lambrichts S, Popleu L, Van Gerven E, Buggenhout S, Bouckaert F. Electroconvulsive therapy during COVID-19-times: our patients cannot wait. Am J Geriatr Psychiatry. 2020;28(7):772-775. doi:10.1016/j.jagp.2020.04.013

2. Espinoza RT, Kellner CH, McCall WV. Electroconvulsive therapy during COVID-19: an essential medical procedure-maintaining service viability and accessibility. $J$ ECT. 2020;36(2):78-79. doi:10.1097/YCT.0000000000000689

3. Tor PC, Phu AHH, Koh DSH, Mok YM. Electroconvulsive therapy in a time of coronavirus disease. $J$ ECT. 2020;36(2):80-85 doi:10.1097/YCT.0000000000000690

4. APA. Practice Guidance for COVID-19. 2020. Available from: https://www.psychiatry.org/psychiatrists/covid-19-coronavirus/prac tice-guidance-for-covid-19. Accessed December 18, 2020.

5. Lapid MI, Seiner S, Heintz H, et al. Electroconvulsive therapy practice changes in older individuals due to COVID-19: expert consensus statement. Am J Geriatr Psychiatry. 2020;28(11):1133-1145. doi:10.1016/j.jagp.2020.08.001

6. Martínez Amorós E, Urretavizcaya M Recomendacines generales de la SEPB para adaptar la práctica de la terapia electroconvulsiva durante la pandemia de COVID-19. https://sepb.es/webnew/wpcontent/uploads/2020/05/TEC-COVID.pdf. 2020. Accessed July 10, 2020.

7. Thiruvenkatarajan V, Dharmalingam A, Armstrong-Brown A, Weiss A, Waite S, Van Wijk R. Uninterrupted anesthesia support and technique adaptations for patients presenting for electroconvulsive therapy during the COVID-19 era. $J$ ECT. 2020;36(3):156-157. doi:10.1097/YCT.0000000000000707

8. Gómez-Arnau J, de Arriba-arnau A, Correas-Lauffer J, Urretavizcaya M. Hyperventilation and electroconvulsive therapy: a literature review. Gen Hosp Psychiatry. 2018;50:54-62. doi:10.1016/j.genhosppsych.2017.09.003

9. Flexman AM, Abcejo AS, Avitsian R, et al. Neuroanesthesia practice during the COVID-19 pandemic: recommendations from Society for Neuroscience in Anesthesiology and Critical Care (SNACC). $J$ Neurosurg Anesthesiol. 2020;32(3):202-209. doi:10.1097/ ANA.0000000000000691

10. Luccarelli J, Fernandez-Robles C, Fernandez-Robles C, et al. Modified anesthesia protocol for electroconvulsive therapy permits reduction in aerosol-generating bag-mask ventilation during the COVID-19 pandemic. Psychother Psychosom. 2020:1-6. doi:10.1159/000509113.

11. International Society for ECT and Neurostimulation (ISEN). COVID19 and ECT. 2020. Available from: https:/isen-ect.org/sites/default/ files/ISEN.COVID19.letter.pdf. Accessed August 11, 2020.

Neuropsychiatric Disease and Treatment

\section{Publish your work in this journal}

Neuropsychiatric Disease and Treatment is an international, peerreviewed journal of clinical therapeutics and pharmacology focusing on concise rapid reporting of clinical or pre-clinical studies on a range of neuropsychiatric and neurological disorders. This journal is indexed on PubMed Central, the 'PsycINFO' database and CAS, and
12. de Arriba-arnau A, Dalmau Llitjos A, Soria V, Menchón JM, Urretavizcaya M. Adaptation of ventilation in ECT sessions during the COVID-19 pandemic. J ECT. 2021;00(00):00. doi:10.1097/ YCT.0000000000000748

13. Gil-Badenes J, Valero R, Valentí M, et al. Electroconvulsive therapy protocol adaptation during the COVID-19 pandemic. J Affect Disord. 2020;276:241-248. doi:10.1016/j.jad.2020.06.051

14. Montero Feijoo A, Maseda E, Adalia Bartolomé R, et al. Practical recommendations for the perioperative management of the patient with suspection or serious infection by coronavirus SARS-CoV. Rev Esp Anestesiol Reanim. 2020;67(5):253-260. doi:10.1016/j. redar.2020.03.003

15. Petrides G, Fink M. The "half-age" stimulation strategy for ECT dosing. Convuls Ther. 1996;12(3):138-146.

16. Martin DM, Gálvez V, Lauf S, et al. The clinical alliance and research in electroconvulsive therapy network. $J$ ECT. 2017;1. doi:10.1097/YCT.0000000000000435

17. Nobler MS, Sackeim HA, Solomou M, Luber B, Devanand DP, Prudic J. EEG manifestations during ECT: effects of electrode placement and stimulus intensity. Biol Psychiatry. 1993;34(5):321-330.

18. $\mathrm{R}$ Core Team. R: A Language and Environment for Statistical Computing [Website]. Vienna, Austria: R Foundation for Statistical Computing; 2020. Available from: https://www.r-project.org/. Accessed March 4, 2020.

19. RStudio Team. RStudio: Integrated Development for $R$ [Website]. Boston, MA: RStudio, PBC; 2020. Available from: http://www.rstu dio.com/. Accessed March 4, 2020.

20. de Arriba-arnau A, Dalmau A, Soria V, et al. Protocolized hyperventilation enhances electroconvulsive therapy. $J$ Affect Disord. 2017;217:225-232. doi:10.1016/j.jad.2017.04.007

21. Kane N, Grocott L, Kandler R, Lawrence S, Pang C. Hyperventilation during electroencephalography: safety and efficacy. Seizure. 2013;23(2):129-134. doi:10.1016/j. seizure.2013.10.010

22. Vidaurre J, Islam MP, Mytinger JR. Pediatric EEG and the COVID-19 pandemic: can we develop a safe transition pathway to reopen our laboratories? J Pediatr Epilepsy. 2020;09(02):029-030. doi:10.1055/s-0040-1712911

23. Surve R, Bansal S, Sriganesh K, Subbakrishna DK, Thirthalli J, Umamaheswara Rao GS. Incidence and risk factors for oxygen desaturation during recovery from modified electroconvulsive therapy: a prospective observational study. $J$ Anaesthesiol Clin Pharmacol. 2015;31(1):99-103. doi:10.4103/09709185.150555JOACP-31-99

24. Koyama Y, Tsuzaki K, Suzuki T, Ozaki M, Saito S. Prevention of oxygen desaturation in morbidly obese patients during electroconvulsive therapy. $J$ ECT. 2020;36(3):161-167. doi:10.1097/ YCT.0000000000000664

25. Aksay SS, Bumb JM, Janke C, Hoyer C, Kranaster L, Sartorius A. New evidence for seizure quality improvement by hyperoxia and mild hypocapnia. J ECT. 2014;30(4):287-291. doi:10.1097/ YCT.0000000000000109 is the official journal of The International Neuropsychiatric Association (INA). The manuscript management system is completely online and includes a very quick and fair peer-review system, which is all easy to use. Visit http://www.dovepress.com/testimonials.php to read real quotes from published authors. 